The prognosis of primary angiosarcoma of the pulmonary system is generally poor, irrespective of whether the location is in the pulmonary parenchyma or the main airway. Angiosarcoma of the pulmonary parenchyma is invariably fatal within months because metastases are usually present at the time of detection, and chemotherapy thus far has not proved effective. The clinical course of solitary angiosarcoma of the main airway is also dismal in previous reports. ${ }^{2,4}$ However, mortality of these patients was mainly caused by massive and intractable hemoptysis of the small resectable tumor rather than by the invasiveness of the disease. From the experience of successful treatment of this patient, angiosarcoma should be considered in the differential diagnosis of a primary tracheal tumor. Furthermore, prompt surgical intervention with adequate resection margins is recommended in the management of these patients when the disease is localized at detection.

\section{References}

1. Spragg RG, Wolf PL, Haghighi P, Abraham JL, Astarita RW. Angiosarcoma of the lung with fatal pulmonary hemorrhage. Am J Med. 1983;74:1072-6.

2. Tuppy H, Kolb R, Eckmayr J. Primary epitheloid angiosarcoma of the proximal trachea. Pathologe. 2000;21:460-3.

3. Sheppard MN, Hansell DM, Du Bois RM, Nicholson AG. Primary epithelioid angiosarcoma of the lung presenting as pulmonary hemorrhage. Hum Pathol. 1997;28:383-5.

4. Palvio DH, Paulsen SM, Henneberg EW. Primary angiosarcoma of the lung presenting as intractable hemoptysis. Thorac Cardiovasc Surg. 1987;35:105-7.

5. Egermayer P. Epidemics of vascular toxicity and pulmonary hypertension: what can be learned? J Intern Med. 2000;247:11-7.

6. Stout AP. Hemangio-endothelioma: a tumor of blood vessels featuring vascular endothelial cells. Ann Surg. 1943;118:445-64.

7. Tsang WY, Chan JK, Fletcher DC. Recently characterized vascular tumors of skin and soft tissues. Histopathology. 1991;19:489-501.

\title{
Primary choriocarcinoma of the lung
}

\author{
Anna Arslanian, MD, ${ }^{a}$ Franco Pischedda, MD, ${ }^{\text {a }}$ Pier Luigi Filosso, MD, ${ }^{\text {a }}$ Paolo Di Marzio, MD, ${ }^{\text {a }}$ Alberto Oliaro, MD, \\ Flavio Fraire, MD, ${ }^{\mathrm{b}}$ and Mauro Papotti, MD, ${ }^{\mathrm{b}}$ Torino, Italy
}

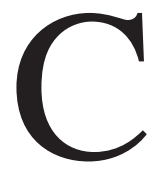

horiocarcinoma is a malignant proliferation of the Langerhans cell and of syncytial cells of trophoblastic origin that is normally situated in the female genital tract after a gestational event such as molar pregnancy, term pregnancy, abortion, or ectopic pregnancy. ${ }^{1}$ Rarely it occurs in either sex as a midline lesion in the retroperitoneum, mediastinum, and the region of the pineal gland. Less frequently it is found in the bladder, liver, stomach, and colon, where it is seen in combination with adenocarcinoma. ${ }^{2-4}$ In men, choriocarcinoma occurs in the testis in combination with such tumors as seminoma, teratoma, and embryonal carcinoma. ${ }^{1}$ The literature contains few cases of lung localization of choriocarcinoma. The prognosis of this tumor is extremely poor, despite surgical and chemotherapeutic treatment. ${ }^{5}$ We report a surgically treated case of choriocarcinoma in a 37-year-old woman who came to our attention because of a lung tumor with radiologic characteristics similar to those of a solitary fibrous tumor of the pleura.

\footnotetext{
From the Department of Thoracic Surgery ${ }^{\mathrm{a}}$ and the Department of Oncology and Biomedical Science, ${ }^{\text {b }}$ University of Torino, Italy.

Received for publication March 15, 2002; accepted for publication April 30, 2002.

Address for reprints: Anna Arslanian, MD, University of Torino, San Giovanni Battista Hospital, Department of Thoracic Surgery, Via Genova, 310126 Torino, Italy (E-mail: slani@interfree.it).

J Thorac Cardiovasc Surg 2003;125:193-6

Copyright $\odot 2003$ by The American Association for Thoracic Surgery $0022-5223 / 2003 \$ 30.00+0$

doi: $10.1067 / \mathrm{mtc} .2003 .121$
}

\section{Clinical Summary}

During surgical evaluation for cholecystectomy for cholelithiasis in a 37-year-old woman, the chest radiograph showed an opacity in the right inferior pulmonary lobe (Figure 1). Computed tomographic scans demonstrated a well-demarcated mass similar to solitary fibrous tumor of the pleura (Figure 2). On physical examination, the woman appeared healthy with stable vital signs. The patient referred in her history to an operation for vesicular mole that had occurred 7 years previously.

A needle biopsy was performed, and the result of cytologic examination of the cells was compatible with the hypothesis of fibrous tumor. A right thoracotomy was performed. The lung appeared to be infiltrated by a tumor not compatible with the hypothesis of a solitary fibrous tumor of the pleura. An inferior lobectomy was performed. The postoperative recovery was uneventful, and the patient was discharged in 8 postoperative days. A definitive histologic examination demonstrated a choriocarcinoma (Figure 3, a-e). $\beta$-Human chorionic gonadotropin ( $\beta$-HCG) was measured in the serum at that time, and the level was high (597 IU/L, whereas the normal level is $0-10 \mathrm{IU} / \mathrm{L}$ ).

Computed tomographic scan of the ovary and the genital apparatus was performed to research a possible primary localization, but nothing was found. Hysteroscopy was also performed, and results were negative. The previous specimen of the vesicular mole was reexamined, but no cells of choriocarcinoma were found.

Chemotherapy was started 2 months after thoracic surgery and consisted of bleomycin, etoposide, and cisplatin. Before the start of chemotherapy, the level of $\beta$-HCG was measured; it was $8 \mathrm{U} / \mathrm{L} 40$ days after surgery. At 1-year follow-up the patient is alive in good condition. The $\beta$-HCG level is actually normal. 


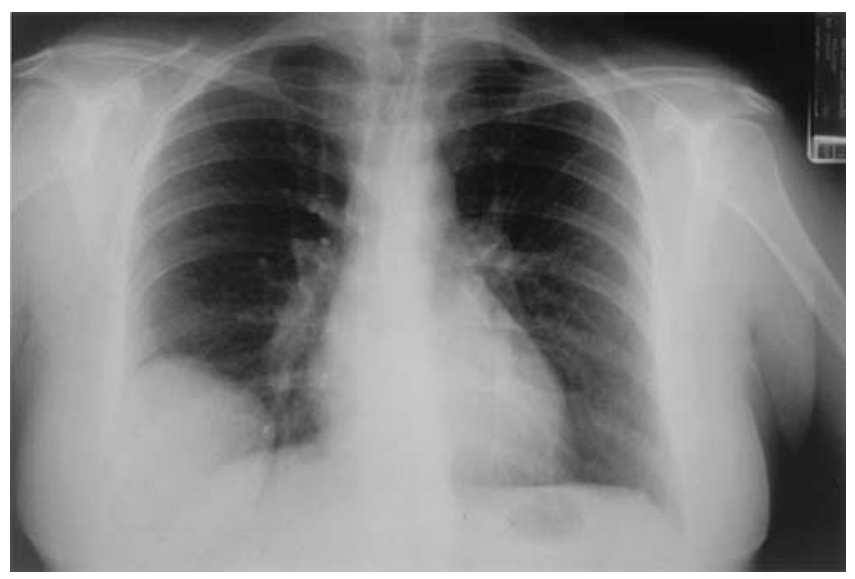

Figure 1. Chest radiograph showing right lower lung mass.

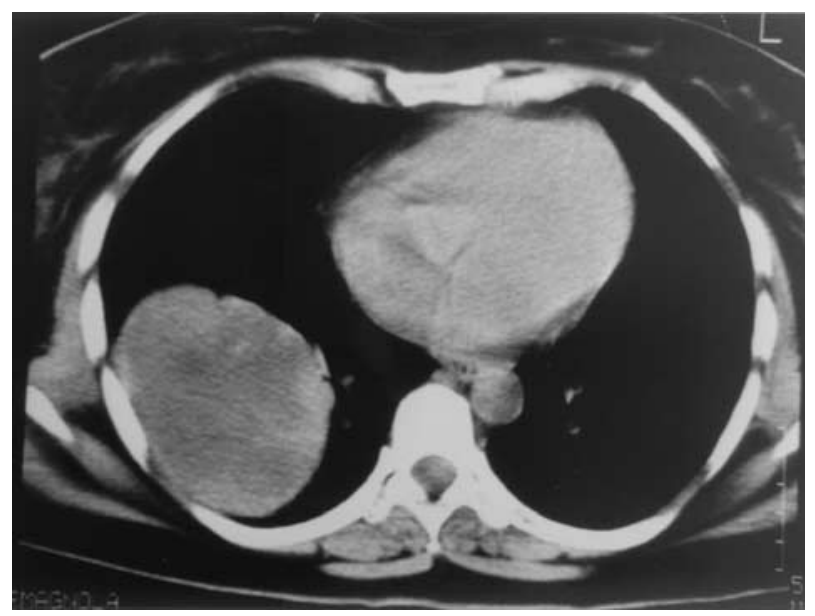

Figure 2. Computed tomographic scan confirming image of welldelimited mass in lung.

\section{Discussion}

Choriocarcinoma is a germ cell tumor containing syncytiotrophoblastic cells and secreting human $\beta$-HCG. ${ }^{4-6}$ Primary choriocarcinoma of the lung is extremely uncommon, with roughly 20 cases reported in the literature. In contrast to other extragonadal germ cell tumors, choriocarcinoma appears more frequently in women and elderly men. The origin of these tumors at this site is unknown, but there are several theories. The tumors could represent metastases from a primary gonadal neoplasm undergoing spontaneous regression. ${ }^{1,6}$ It is also possible that they are derived from a progression to malignancy of trophoblastic emboli from a previous vesicular mole, term pregnancy, abortion, or ectopic pregnancy months or years before. ${ }^{3,6}$ Another hypothesis is that there was a differentiation or metaplasia to trophoblast from a primary lung cancer. Finally, the tumor could have been generated from remaining germ cells after an anomalous migration during the embryonic phase. ${ }^{6}$

Pulmonary choriocarcinoma can appear in different ways. Recurrent hemoptysis seems to be the most frequent symptom; in addition to this, it has been reported as chest pain, persistent cough, and signs of feminization in men with gynecomastia, loss of libido, and testicular atrophy. Distant metastases are commonly present in the lung, brain, and kidney. ${ }^{3,6}$

Two typical characteristics of this tumor represent the choriocarcinoma syndrome: the bleeding tendency of the primary and metastatic sites and the constant elevation of serum $\beta$-HCG levels. ${ }^{6} \beta$-HCG is useful for diagnostic, prognostic, and follow-up purposes. $^{2}$

A positive $\beta$-HCG test result in patients with hemoptysis and progressive dyspnea could be diagnostic for a pulmonary choriocarcinoma and may be helpful for a early diagnosis.5,6 The diagnostic criteria would include lack of a previous gynecologic malignancy, solitary or predominant lung lesion with the exclusion of a gonadal primary site, raised serum $\beta$-HCG titers that become normal after surgery or chemotherapy, and pathologic confirmation of the disease.

Hematologic analysis shows also leukocytosis with neutrophilia and elevated sedimentation rate. ${ }^{5}$ Although urinary $\beta$-HCG has considerable diagnostic and prognostic value, a negative test result does not exclude presence of these neoplasms.

The radiologic presentation may be nodular image, diffuse shadowing, or miliary or micronodular forms with or without interstitial infiltrates. ${ }^{5}$ Diagnosis is often delayed, and pleural effusion may be present.

Bronchoscopic examination yields negative results for this tumor. ${ }^{5}$ Percutaneous needle biopsy often shows a largely necrotic nonBsmall cell carcinoma interpreted as squamous cell type from the deeply eosinophilic cytoplasm, suggesting keratinization in the few viable anaplastic cells. Electron microscopic features of tonofilaments are present on ultrastructural analysis, also in keeping with squamous differentiation. Immunohistochemical staining shows cytotrophoblastic cells reactive for $\beta$-HCG. Coexistence with adenocarcinoma of the lung has been reported in the literature. ${ }^{1,3}$

The surgical option is often selected on the basis of presumed squamous cell carcinoma of the lung in the absence of distant and regional metastases, and the correct diagnoses represent a surprise at histologic diagnosis. ${ }^{2}$ So, as in this case, $\beta$-HCG was not measured before surgery. The natural course of choriocarcinoma is rapidly fatal in the great majority of cases. ${ }^{5}$ We think that surgical excision according to the oncologic criteria for bronchopulmonary cancer is advisable, however, because there are no established therapeutic guidelines and this tumor evolved rapidly with bleeding, chest pain, and dyspnea. More or less 30 days after surgery, the $\beta$-HCG level returned to normal. During follow-up an elevation of $\beta$-HCG would be indicative of micrometastasis. ${ }^{2}$

Chemotherapy is indicated for this tumor independently of surgery and consists of bleomycin, etoposide, and cisplatin. ${ }^{2}$ Nongestational choriocarcinoma, unlike that arising from a gestational tumor, is unresponsive to irradiation. The reason is unknown. ${ }^{1}$ 

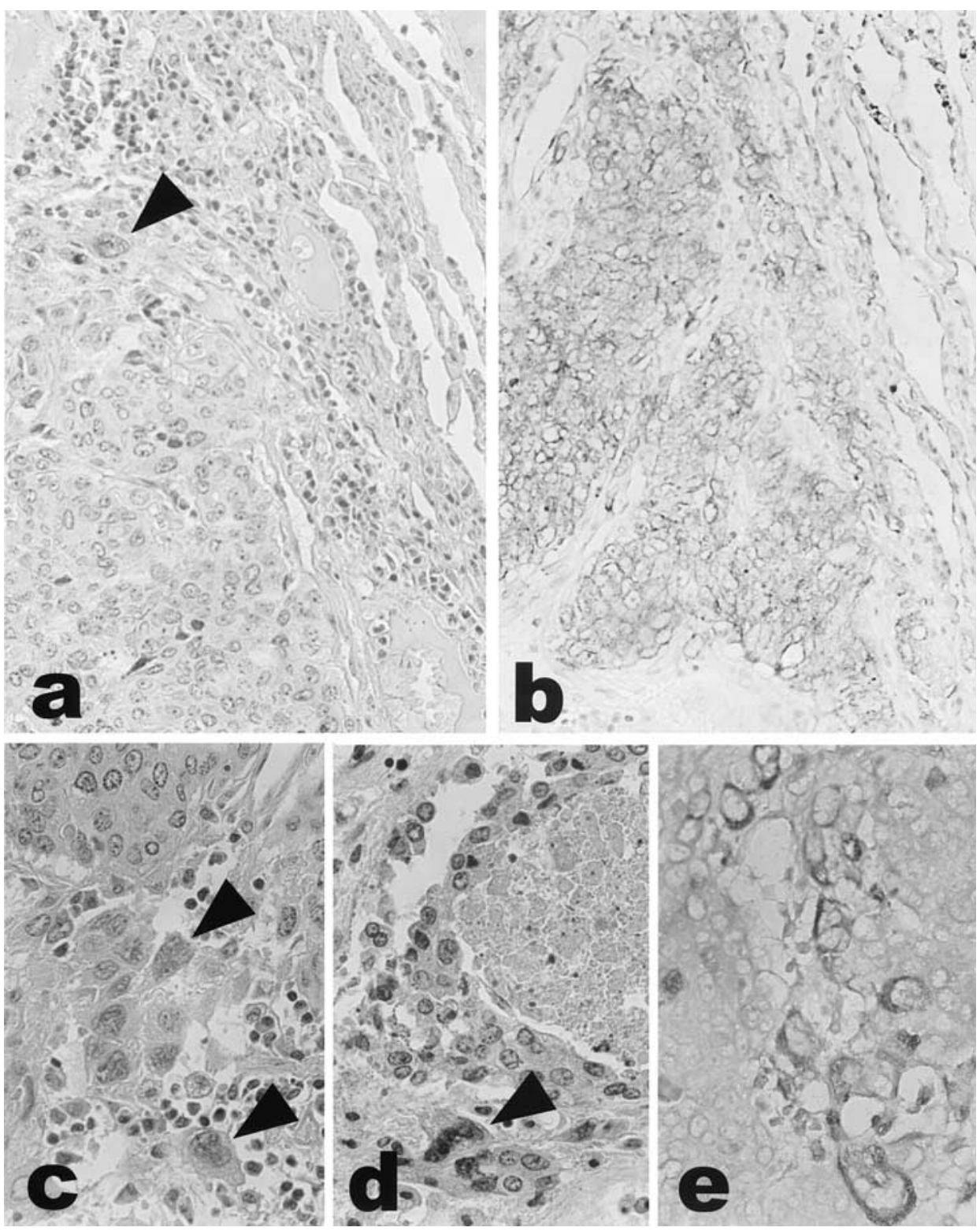

Figure 3. a, Neoplastic giant cells at lung parenchyma (hematoxylin-eosin, original magnification $\times 200$ ). $b$, Immunohistochemical staining for $\beta$-HCG: evidence of positivity of neoplastic giant cells (original magnification $\times 200$ ). $c$ and $d$, Detail of choriocarcinoma: observation of pathologic plurinucleate giant cells (hematoxylin-eosin, original magnification $\times 400$ ). e, Immunohistochemical staining for $\beta$-HCG: nidi of cells positive for $\beta$-HCG (original magnification $\times 400$ ).

\section{Frequent Signs and Symptoms}

\section{General syndrome.}

- Significant deterioration of the patient's health is seen, as in cancer.

- There is scanty but recurrent hemoptysis.

- Patient reports chest pain.

- Patient has a dry, persistent cough
- Signs of feminization are present in men, with gynecomastia, loss of libido, and testicular atrophy.

Radiologic evidence. Appearance of the tumor may be diffuse or defined.

Hematologic analysis. Positive results are seen on $\beta$-HCG test. Leukocytosis is present, with neutrophilia and elevated sedimentation rate.

Urinalysis. $\beta$-HCG may be present in the urine. 


\section{References}

1. Canver CC, Voytovich MC. Resection of an unsuspected primary pulmonary choriocarcinoma. Ann Thorac Surg. 1996;61:1249-51.

2. Sridar KS, Saldana MJ, Thurer RJ, Beattie EJ. Primary choriocarcinoma of the lung: report of a case treated with intensive multimodality therapy and review of the literature. J Surg Oncol. 1989; 41:93-7.

3. Chen F, Tatsumi A, Numoto S. Combined choriocarcinoma and adenocarcinoma of the lung occurring in a man: a case report and review of the literature. Cancer. 2001;91:123-9.
4. Tanimura A, Natsuyama H, Kawano M, Tanimura Y, Tanaka T, Kitazono M. Primary choriocarcinoma of the lung. Hum Pathol. 1985;16:1281-4.

5. Zapatero J, Bellon J, Baamonde C, Aragoneses FG, Cubillo J, Orusco E, et al. Primary choriocarcinoma of the lung: presentation of a case and review of the literature. Scand J Thorac Cardiovasc Surg. 1982; 16:279-81.

6. Aparicio J, Oltra A, Martinez-Moragon E, Llorca C, Gomez-Aldaravi L, Pastor M. Extragonadal nongestational choriocarcinoma involving the lung: a report of three cases. Respiration. 1996;63:251-3.

\title{
Brucella melitensis endocarditis of ventricular septal defect patch: Successful surgical treatment without replacement
}

\author{
Ugursay Kiziltepe, MD, ${ }^{a}$ Adnan Uysalel, MD, ${ }^{a}$ Ercan Tutar, MD, ${ }^{\text {b }}$ and Hakki Akalin, MD, ${ }^{a}$ Ankara, Turkey
}

B rucella melitensis endocarditis is a rare but fatal complication of brucellosis. It is usually located on native and prosthetic valves. ${ }^{1}$ Endocarditis of a prosthetic ventricular septal defect (VSD) patch is rare. Only one case of treatment by means of replacement of the prosthetic patch and antibiotic administration has been reported. ${ }^{2}$ When present, removal and replacement of the infected tissue or prosthetic material has been recommended in addition to long-term antibiotic treatment to achieve a cure. ${ }^{3,4}$ Replacement of the prosthetic patch might not always be necessary, as was the case in our experience.

\section{Clinical Summary}

A 12-year-old boy with cyanosis and growth retardation was given a diagnosis of tetralogy of Fallot. He underwent a successful total correction operation with Dacron patch repair of a VSD with interrupted pledgeted sutures and transannular Dacron patch right ventricular outflow tract reconstruction. The early postoperative period was uneventful, and no residual VSD was found at early postoperative echocardiography. He was discharged to home, but 2 weeks later, he was readmitted to the hospital with fever and arthralgia. The initial echocardiography revealed a very small hole at the edge of the VSD patch and no apparent vegetation. At that time, blood cultures did not show growth of any microorganism. Wide-spectrum antibiotics and antipyretic treatment were started. The erythrocyte sedimentation rate was $95 \mathrm{~mm} / \mathrm{h}$, and the C-reactive protein level was $3+$. After 1 week, his general status had

From the Departments of Cardiovascular Surgery, Cardiac Center, ${ }^{\mathrm{a}}$ and Pediatric Cardiology, ${ }^{\mathrm{b}}$ Ankara School of Medicine, Ankara, Turkey.

Received for publication March 1, 2002; accepted for publication April 2, 2002.

Address for reprints: Ugursay Kiziltepe, MD, Sokollu Cad. Nakis Sok., 8/14 Dikmen, Ankara 06460, Turkey (E-mail: uk9316@hotmail.com).

J Thorac Cardiovasc Surg 2003;125:196-7

Copyright $\odot 2003$ by The American Association for Thoracic Surgery

$0022-5223 / 2003 \$ 30.00+0$

doi: $10.1067 / \mathrm{mtc} .2003 .114$ worsened, and symptoms of congestive heart failure and a harsh pansystolic murmur had appeared. Repeat echocardiography showed the presence of a large recurrent VSD but no visible vegetation (Figure 1). Cardiac catheterization revealed a pulmonary/systemic flow ratio of 2.2 and a pulmonary artery pressure of $45 \mathrm{~mm} \mathrm{Hg}$. His culture results were still negative. He received vancomycin and amikacin treatments for 10 days before surgical repair. During reoperation, a large hole and extensive vegetation were found at the muscular septal edge of the prosthetic patch. The pledget-supported sutures were pulled off through the torn myocardium. Because the general status of the patient was poor and he might not tolerate long crossclamp and cardiopulmonary bypass (CPB) periods and because the presence of $B$ melitensis endocarditis was not known at this stage, we decided to perform extensive debridement of the vegetation and reattachment of the same patch to the septum instead of resection and replacement of the prosthetic patch. After reattachment of the patch with 4 pledgeted sutures, the heart was deaired, and the crossclamp was removed. After rewarming, the patient was easily weaned from CPB. Crossclamp and CPB times were 46 and 71 minutes, respectively. The postoperative period was uneventful. Vancomycin and amikacin treatments were continued for another 10 days. After the cultures from the debrided tissue and blood revealed $B$ melitensis endocarditis, those antibiotics were replaced with $200 \mathrm{mg} / \mathrm{d}$ doxycycline and $600 \mathrm{mg} / \mathrm{d}$ rifampin (INN: rifampicin), which were continued for 4 months. No complications were seen during the postoperative period. After antibiotic treatment was completed, the patient was totally symptom free. He has completed his second postoperative year without additional antibiotic treatment, and his blood culture and serologic test results are negative. His most recent echocardiography at 24 months postoperatively showed an intact VSD patch without vegetation or residual VSD. He is considered to be totally cured.

\section{Comment}

Brucellosis is seen more frequently in Mediterranean, Arabic peninsula, Indian subcontinent, and South-Central American regions. It is a zoonosis and is transmitted to human patients by means of 